\title{
GROUPS WHOSE PROPER QUOTIENTS ARE HYPERCENTRAL
}

\author{
L. A. KURDACHENKO and I. Y. SUBBOTIN
}

(Received 19 December 1997; revised 27 June 1998)

Communicated by J. R. J. Groves

\begin{abstract}
Groups, all proper factor-groups of which are hypercentral of finite torsion-free rank, are studied in this article.
\end{abstract}

1991 Mathematics subject classification (Amer: Math. Soc.): primary 20F19, $20 \mathrm{~F} 14$.

\section{Introduction}

Let $G$ be a group and let $N$ be a normal subgroup of $G$. The factor-group $G / N$ is said to be a proper factor-group if $N \neq\langle 1\rangle$. The influence of properties of proper factor-groups on properties of groups was the subject of investigation of many authors. The classic example in this area is the following theorem of Robinson [14, Theorem 10.51]: a finitely generated soluble group is nilpotent if all its finite factor-groups are nilpotent. The set of all finite factor-groups also plays an important role in the study of finitely presented groups and in algorithmic problems. The influence of the structure of torsion factor-groups on the structure of some soluble groups has been studied in [21]. But if we consider all proper factor-groups, the influence of their structure on the structure of a group will increase powerfully.

Let $\mathfrak{I}$ be a class of groups. A group $G$ is called a just-non- $\mathfrak{I}$-group if $G \notin \mathfrak{I}$, but every proper factor-group of $G$ belongs to $\mathfrak{I}$. The structure of just-non-I-groups has already been studied for several choices of the class $\mathfrak{I}$. The first research on this topic was done by Newman [11, 12], who considered just-non-abelian groups. Later, the class of just-non- $\mathfrak{I}$-groups was investigated in the cases where $\mathfrak{I}$ is chosen to be the class of finite groups $[9,10,18]$, of polycyclic or supersoluble groups $[5,16]$, of

(C) 1998 Australian Mathematical Society $0263-6115 / 98 \$ A 2.00+0.00$ 
Chernikov groups [1], of groups with transitive normality [15], of finite-by-abelian or central-by-finite groups [17]. Franciosi and de Giovanni considered groups, all proper factor-groups of which are nilpotent of class $\leq c$ [2]. Some generalization of this situation can be found in [22]. If $G$ is a non-monolithic group in which all proper factor-groups are nilpotent of class $\leq c$, then $G$ is nilpotent also. But if we reject the bounding of class of nilpotency, the non-monolithic case will be much more complicated. Groups, all proper factor-groups of which are hypercentral of finite 0 rank (torsion-free rank), are studied in this paper. Note that every simple group $G \notin \mathfrak{I}$ is a just-non-I-group. Therefore, in an investigation concerning just-non-I-groups, it is natural to consider groups which include a non-identity abelian normal subgroup, that are groups with a non-identity Fitting subgroup.

The main results of our paper are the following theorems.

THEOREM 1. Let $G$ be a non-monolithic group, all proper factor-groups of which are hypercentral groups of finite 0-rank. If Fitt $G \neq\langle 1\rangle$, then $G$ is hypercentral. In particular, if every proper factor-group of a non-monolithic group $G$ is periodic and hypercentral, then $G$ is hypercentral.

THEOREM 2. Let $G$ be a monolithic group with Fitt $G \neq\langle 1\rangle$. Then $G$ is a just-nonhypercentral group if and only if $G$ satisfies the following conditions:

(1) Fitt $G=M$ is the monolith of $G$ (in particular, $M$ is abelian);

(2) $M$ is a maximal abelian normal subgroup of $G$;

(3) $G=M \lambda H$ where $H=N_{G}(H)$ is a hypercentral group (we use $\lambda$ to denote the semidirect product with the normal subgroup $M$ );

(4) all complements to $M$ are conjugate in $G$;

(5) the periodic part $T$ of the center $\zeta(H)$ is locally cyclic;

(6) if $M$ is an elementary abelian $p$-group for some prime $p$ then $T$ is a $p^{\prime}$-group.

Moreover, if every proper factor-group of $G$ has a finite 0-rank, then $M$ is an elementary abelian p-subgroup and $T=\zeta(H)$. In particular, if every proper factorgroup of $G$ is periodic, then $G$ is also periodic.

\section{Some preliminary results}

LEMMA 1.1. Let $G$ be a just-non-hypercentral group. Then

(1) $G$ does not include normal non-identity subgroups $R_{1}$ and $R_{2}$ such that $R_{1} \cap R_{2}=$ $\langle 1\rangle$;

(2) $\zeta(G)=\langle 1\rangle$

(3) if $G$ includes a finite non-identity normal subgroup $F$, then $G$ is finite. 
PROOF. (1) From $R_{1} \cap R_{2}=\langle 1\rangle$ we obtain the imbedding $G \leq G / R_{1} \times G / R_{2}$, which shows that $G$ is hypercentral.

(2) is obvious.

(3) Suppose that $G$ is infinite. We can assume that $F$ is a finite minimal normal subgroup of $G$. From (1) we obtain that $F \leq C_{G}(F)$, in particular $F$ is abelian. The factor-group $G / C_{G}(F)$ is finite, so $C_{G}(F)=C$ is infinite. Since $G / F$ is hypercentral, $C / F \cap \zeta(G / F) \neq\langle 1\rangle$. Let $F \neq a F \in C / F \cap \zeta(G / F), A=\langle F, a\rangle$. If $a$ is an element of an infinite order then $A^{k}=\left\langle a^{k}\right\rangle \neq\langle 1\rangle$ for $k=|F|$. Then $A^{k} \cap F=\langle 1\rangle$ and we have a contradiction of (1). If $|a|$ is finite then $A$ is finite, in particular, $A$ satisfies Min- $G$. Since $G / A$ is hypercentral, by [19, Theorem $\left.1^{\prime}\right] A$ has the decomposition $A=A_{1} \times A_{2}$ where $A_{1}$ and $A_{2}$ are $G$-invariant subgroups such that every $G$-chief factor of $A_{1}$ is $G$-central and every $G$-chief factor of $A_{2}$ is not $G$-central. Since $A \neq F$ and $A / F \leq \zeta(G / F), A_{1} \neq\langle 1\rangle$. This means that $\zeta(G) \neq\langle 1\rangle$, and we have a contradiction of (2).

LEMMA 1.2. Let $G$ be an infinite just-non-hypercentral group and let $A$ be a maximal normal abelian subgroup of $G$. Assume that $A \neq\langle 1\rangle$. Then

(1) either $A$ is an infinite elementary abelian p-subgroup for some prime $p$, or $A$ is a torsion-free subgroup;

(2) $A=C_{G}(A)$;

(3) if $A \neq z A \in \zeta(G / A)$, then $C_{G}(z)=\langle 1\rangle$.

Proof. (1) Let $T$ be the periodic part of $A$. Assume that $T \neq\langle 1\rangle$. Lemma 1.1 yields that $T$ is a $p$-subgroup for some prime $p$. Put $T_{1}=\Omega_{1}(T)=\{x \in T \mid$ $\left.x^{p}=1\right\}$. Lemma 1.1 implies that $T_{1}$ is an infinite elementary abelian $p$-subgroup. Suppose that $T \neq T_{1}$. Since $G / T_{1}$ is hypercentral, $T / T_{1} \cap \zeta\left(G / T_{1}\right) \neq\langle 1\rangle$. Let $T_{1} \neq c T_{1} \in T / T_{1} \cap \zeta\left(G / T_{1}\right)$, then $[c, g] \in T_{1}$ for each $g \in G$. It follows that $1=[c, g]^{p}=\left[c^{p}, g\right]$. Since $c \notin T_{1}, c^{p} \neq 1$. This means that $\zeta(G) \neq\langle 1\rangle$. However, this contradicts Lemma 1.1. Hence $T=T_{1}$.

If $A \neq T$, then $A=T \times B$ for some subgroup $B$ (see, for example, [3, Theorem 27.5]). In particular, $A^{p}$ is a non-identity $G$-invariant torsion-free subgroup. But this contradicts Lemma 1.1. Consequently, if $T \neq\langle 1\rangle$ then $A$ is elementary abelian.

(2) is almost obvious.

(3) Consider the mapping $\varphi: A \rightarrow A$ defined by the rule $a \varphi=[a, z], a \in A$. Since $z A \in \zeta(G / A), \varphi$ is a $G$-endomorphism of $A$. In particular, $\operatorname{Im} \varphi=[A, z]$ and $\operatorname{Ker} \varphi=$ $C_{A}(z)$ are $G$-invariant subgroups of $A$. By $(2) z \notin C_{G}(A)$ so that $C_{A}(z) \neq A$. Suppose that $C_{A}(z) \neq\langle 1\rangle$. Then $G / C_{A}(z)$ is hypercentral and $\zeta\left(G / C_{A}(z)\right) \cap A / C_{A}(z) \neq\langle 1\rangle$. Let $C_{A}(z) \neq a C_{A}(z) \in \zeta\left(G / C_{A}(z)\right) \cap A / C_{A}(z)$. Since $a \notin C_{A}(z), a_{1}=[a, z] \neq 1$. Let $g$ be an arbitrary element of $G$. Rewrite the Hall-Witt identity in the form

$$
[[a, z], g]^{x}\left[\left[z^{-1}, g^{-1}\right], a\right]^{g}\left[\left[g, a^{-1}\right], z^{-1}\right]^{a}=1, \quad x=z^{-1} .
$$


Since $g A \in \zeta(G / A),\left[z^{-1}, g^{-1}\right] \in A$, so that $\left[\left[z^{-1}, g^{-1}\right], a\right]=1$. Since $a C_{A}(z) \in$ $\zeta\left(G / C_{A}(z)\right),\left[g, a^{-1}\right] \in C_{A}(z)$, and $\left[\left[g, a^{-1}\right], z^{-1}\right]=1$. It follows that $[[a, z] g]=1$, that is $1=\left[a_{1}, g\right]$. This means that $\zeta(G) \neq\langle 1\rangle$. This contradicts Lemma 1.2, so (3) is proved.

Recall the definition of finite 0-rank.

DEFINITION. We say that a group $G$ has finite 0-rank (or finite torsion-free rank) which is equal to $r$, if $G$ has a finite subnormal series $\langle 1\rangle=G_{0}<G_{1}<\cdots<G_{n}=$ $G, r$ factors of which are infinite cyclic groups, and all remaining factors are torsion groups.

We will denote the 0 -rank of group $G$ by $r_{0}(G)$.

LEMMA 1.3. Let $G$ be a torsion-free nilpotent group of finite 0-rank and let $p$ be a prime number. Then $G$ has a finite subnormal series $\langle 1\rangle=H_{0}<H_{1}<H_{2}<\cdots<$ $H_{n}=G$, in which every factor $H_{i+1} / H_{i}$ is torsion and p-divisible, $1 \leq i \leq n-1$, and the subgroup $H_{1}$ is finitely generated.

PROOF. Since the factor-group of a torsion-free nilpotent group over its center is torsion-free also (see, for example, [13, Theorem 2.25]), we can use induction on the class of nilpotence $c$ of the group $G$. If $c=1$ then $G$ is an abelian torsion-free group of finite 0-rank. Let $\left\{a_{i} \mid 1 \leq i \leq r\right\}$ be a maximal $Z$-independent subset of $G$, $B=\left\langle a_{i} \mid 1 \leq i \leq r\right\rangle$. Then $G / B$ is a torsion abelian group of finite Prufer rank, and therefore its Sylow $p$-subgroup $P / B$ is a Chernikov group. Then $P / B$ includes the finite subgroup $H / B$ such that $P / H$ is a divisible Chernikov $p$-group. In this case $G / H$ is a $p$-divisible group.

Let $c>1$ and $C=\zeta(G)$. Then $G / C$ is a torsion-free nilpotent group of class $c-1$, and by the induction hypothesis $G / C$ has a finite subnormal series $C=H_{2}<$ $H_{3}<\cdots<H_{n}=G$ such that $H_{2} / C$ is finitely generated and all remaining factors $H_{i+1} / H_{i}$ are torsion and $p$-divisible, $2 \leq i \leq n-1$. Since $H_{2} / C$ is finitely generated, $H_{2}=F \cdot C$ for some finitely generated subgroup $F$. Since $C=\zeta(G), F$ is normal in $H_{2}$. By the induction hypothesis $C$ includes the finitely generated subgroup $D$ such that $C / D$ is torsion and $p$-divisible. Put $H_{1}=D \cdot F$. Then $H_{1}$ is finitely generated and normal in $H_{2}$, and $H_{2} / H_{1}=C F / D F=C D F / D F \cong C / C \cap D F=C / D(C \cap F)$, so that $H_{2} / H_{1}$ is torsion and $p$-divisible.

LEMMA 1.4. Let $F$ be a field, $G$ a hypercentral group, and let $A$ be an $F G$ module. Suppose that $A$ includes an $F G$-submodule $B$ satisfying the following conditions:

(1) $A(x-1) \leq B$ for every $x \in G$; 
(2) B is a simple FG-submodule;

(3) $C_{G}(B) \neq G$.

Then $A$ includes an $F G$-submodule $C$ such that $A=B \oplus C$.

PROOF. We can assume that $C_{G}(A)=\langle 1\rangle$. Let $1 \neq z \in \zeta(G)$. Then the mapping $\varphi: a \rightarrow a(z-1), a \in A$, is an $F G$-endomorphism and $\operatorname{Ker} \varphi=\operatorname{Ann}_{A}(z-1)=C_{A}(z)$, $\operatorname{Im} \varphi=A(z-1)$. It follows from (1) that $A(z-1) \leq B$. Since $B$ is a simple $F G$ submodule, $A(z-1)=B$. If we assume that $B(z-1)=\langle 0\rangle$, then we have $B \leq \operatorname{Ker} \varphi$, therefore $B=A(z-1) \cong{ }_{F G} A / \operatorname{Ker} \varphi$. But in this case, $B(x-1)=\langle 0\rangle$ for any $x \in G$. This is a contradiction of condition (3). Hence $A(z-1)=B(z-1)$. It follows that $A=\operatorname{Ann}_{A}(z-1)+B$. Since $B=B(z-1), \operatorname{Ann}_{A}(z-1) \cap B=\langle 0\rangle$ so that $A=B \oplus C$ where $C=\operatorname{Ann}_{A}(z-1)$.

DEFINITION. Let $G$ be a just-non-hypercentral group, $A$ a non-identity normal abelian subgroup of $G, \mathscr{R}_{G}(A)=\{B \mid B$ is a non-identity $G$-invariant subgroup of $A\}$. Let $M=\cap \mathscr{R}_{G}(A)$. Then either $M=\langle 1\rangle$ (non-monolithic case) or $M \neq\langle 1\rangle$. In the second case $M$ is called the monolith of group $G$.

Lemma 1.4 implies that either $A$ is an elementary abelian $p$-group or $A$ is torsionfree. Consequently, we must consider the following situations: non-monolithic case of characteristic $p$, non-monolithic case of characteristic 0 , and the monolithic case.

\section{Non-monolithic case of characteristic $p$}

Everywhere in this section (except Proposition 2.4), $G$ is a just-non-hypercentral non-monolithic group and $A$ is a maximal normal abelian subgroup of $G$. We also assume that $A$ is an elementary abelian p-group for some prime $p$. Lemma 1.1 implies that $A$ is infinite.

LEMMA 2.1. The factor-group $G / A$ is torsion-free. In particular, if every proper factor-group of $G$ has finite 0-rank, then $G / A$ is a nilpotent torsion-free group of finite 0-rank.

ProOf. Let $P / A$ be a Sylow $p$-subgroup of $G / A$. Suppose that $P / A$ is a nonidentity. Then $P / A \cap \zeta(G / A) \neq\langle 1\rangle$. Let $g A \neq A, g A \in P / A \cap \zeta(G / A)$. Then $g$ is a $p$-element and the subgroup $\langle g, A\rangle$ is nilpotent (see, for example, [14, Lemma 6.34]). It follows that $C_{A}(g) \neq\langle 1\rangle$. However, this is a contradiction of Lemma 1.2.

Let $Q / A$ be a Sylow $p^{\prime}$-subgroup of $G / A$. Suppose that $Q / A \neq\langle 1\rangle$. Then $\langle 1\rangle \neq$ $R / A=Q / A \cap \zeta(G / A)$. Let $B \in \mathscr{R}_{G}(A)$. Since $G / B$ is hypercentral and $A / B$ is the Sylow $p$-subgroup of $R / B, R / B=A / B \times S / B$ where $S / B$ is a Sylow $p^{\prime}$-subgroup 
of $R / B$. Since $S / B \cong R / A, R / B$ is abelian so that $[R, R] \leq \cap \mathscr{R}_{G}(A)=\langle 1\rangle$. Thus $R$ is an abelian normal subgroup of $G$. But $A \leq R$ and $A \neq R$, so we obtain a contradiction with the choice of $A$. This contradiction shows that $G / A$ is torsion-free.

If $G / A$ has a finite 0 -rank, then it is nilpotent (see, for example, [14, Theorem $6.36])$.

Now we need some module-theoretical concepts.

Definition. Let $J$ be a principal ideal domain, $A$ a $J$-module, $a \in A$, and let $\operatorname{Ann}_{J}(a)=\{x \in J \mid a x=0\}$. An element a is called $J$-torsion if $\operatorname{Ann}_{J}(a) \neq\langle 0\rangle$. The set $t_{J}(A)$ of all $J$-torsion elements of $A$ is a $J$-submodule of $A$. The submodule $t_{J}(A)$ is called the $J$-torsion part of $A$. If $A=t_{J}(A)$ then $A$ is called the $J$-torsion module. If $t_{J}(A)=\langle 0\rangle$, then we say that $A$ is $J$-torsion-free.

Let $I$ be an ideal of $J$. Put $A_{l}=\left\{a \in A \mid a I^{n}=\langle 0\rangle\right.$ for some $\left.n \in N\right\}$. It is easy to see that $A_{l}$ is a $J$-submodule of $A$. This $J$-submodule is called the $I$-component of module $A$. Let $\operatorname{Spec}(J)$ be the set of all maximal ideals of $J$. If $a \in t_{J}(A)$, then $\operatorname{Ann}_{J}(a)=P_{1}^{k_{1}} \ldots P_{l}^{k_{l}}$ for some $P_{1}, \ldots, P_{l} \in \operatorname{Spec}(J), k_{1}, \ldots, k_{l} \in N$. Put $\Pi_{J}(a)=\left\{P_{1}, \ldots, P_{k}\right\}, \Pi_{J}(A)=\cup_{a \in t(A)} \Pi_{J}(a)$.

As in the case when $J=Z$, we can prove that $t_{J}(A)=\oplus_{P \in \Theta} A_{P}, \Theta=\Pi_{J}(A)$.

We can consider $A$ as $Z H$-module where $H=G / A$ is a hypercentral group.

Lemma 2.2. (1) Let $A \neq x A \in \zeta(G / A)$, then $A$ (as $F_{p}\langle x\rangle$-module) is $F_{p}\langle x\rangle$ torsion-free.

(2) If $B$ is a non-identity $G$-invariant subgroup of $A$, then $C_{G}(B)=A$.

PROOF. (1) Since $|x A|$ is infinite, by Lemma $2.1,|x|$ is infinite too and $F_{p}\langle x\rangle$ is a principal ideal domain. We consider $A$ as $F_{p} H$-module where $H=G / A$ and use additive notation for $A$. Let $T$ be the $F_{p}\langle x\rangle$-torsion part of $A$ and suppose that $T \neq\langle 0\rangle$. Since $x A \in \zeta(G / A)$, the $I$-component of $A$ is a $F_{p} H$-submodule for every ideal $I$ of $F_{p}\langle x\rangle$. Lemma 1.1 yields that $\Pi_{J}(A)=\{P\}$ for some $P \in \operatorname{Spec}\left(F_{p}\langle x\rangle\right)$. Put $T_{1}=\{a \in T \mid a P=\langle 0\rangle\}$ and assume that $T \neq T_{1}$. The factor-group $G / T_{1}$ is hypercentral, therefore, $\zeta\left(G / T_{1}\right) \cap T / T_{1} \neq\langle 1\rangle$. Let $a T_{1} \neq T_{1}, a T_{1} \in T / T_{1} \cap \zeta\left(G / T_{1}\right)$. Then $\operatorname{Ann}_{F_{p}\langle x\rangle}\left(a T_{1}\right)=(x-1) F_{p}\langle x\rangle=P_{1}$. Since $\Pi_{F_{p}(x)}(T)=\Pi_{F_{p}(x)}\left(T / T_{1}\right), P=P_{1}$. However, in this case, $\operatorname{Ann}_{F_{p}\langle x\rangle}\left(T_{1}\right)=P_{1}$. In other words, $T_{1} \leq \operatorname{Ann}_{A}(x-1)=C_{A}(x)$, which is a contradiction of Lemma 1.2. Hence $T=T_{1}$.

Suppose that $T \neq A$. As in the abelian groups case, we can prove that $A=T \oplus C$ for some $F_{p}\langle x\rangle$-submodule $C$. Let $B=A P$. Then $B=C P \leq C$, in particular, $B \cap C=\langle 0\rangle$. Since $x A \in \zeta(G / A), B$ is a $G$-invariant subgroup of $A$ and we obtain a contradiction of Lemma 1.1. Hence $A=T$. Since $F_{p}\langle x\rangle$ is a principal ideal domain, there is an element y such that $P=y F_{p}\langle x\rangle$. Since $P$ is a maximal ideal of $F_{p}\langle x\rangle, y$ 
is an irreducible polynomial in $x$. Let $a \in A$. From $a F_{p}\langle x\rangle \cong F_{p}\langle x\rangle / P$ we obtain $\left|a F_{p}\langle x\rangle\right|=\left|\langle a\rangle^{\langle x\rangle}\right|=p^{t}$ where $t=\operatorname{deg} y$. It follows that $x^{l} \in C_{G}(a)$ where $l=\left(p^{t}\right)$ !. Since it is true for each $a \in A, x^{l} \in C_{G}(A)$. By Lemma 1.2 $C_{G}(A)=A$. This means that $|x A|$ has finite order. However, Lemma 2.1 implies that $G / A$ is torsion-free. Hence $T=\langle 0\rangle$.

(2) It follows from the choice of $B$ that $G / B$ is hypercentral. If $a \in A$, then the subgroup $\langle a B, x B\rangle$ is nilpotent. It follows that

$$
[a, \underbrace{x, \ldots, x}_{n}] \in B \quad \text { for some } \quad n \in N .
$$

We can rewrite it using the additive notation: $a(x-1)^{n} \in B$. This means that the factor-module $A / B$ is $F_{p}\langle x\rangle$-torsion. Let $g \in C_{G}(B), a g=a_{1}$, then

$$
a_{1}(x-1)^{n}=a g(x-1)^{n}=a(x-1)^{n} \cdot g=a(x-1)^{n}, \text { or }\left(a_{1}-a\right)(x-1)^{n}=0 .
$$

Since $A$ is $F_{p}\langle x\rangle$-torsion-free, this means that $a-a_{1}=0$, that is $a g=a$. In other words, $g \in C_{G}(A)=A$.

DEFINITION. Let $H$ be a group and let $A$ be a $Z H$-module. Then $A$ is called the just infinite $Z H$-module, if $A$ satisfies the following conditions:

(JI 1) if $B$ is a non-zero $Z H$-submodule of $A$, then $A / B$ is finite;

(JI 2) $\cap\{B \mid B$ is a non-zero $Z H$-submodule of $A\}=\langle 0\rangle$.

LEMMA 2.3. Suppose that all proper factor-groups of $G$ have finite 0 -rank. Let $1 \neq a \in A$, and $B=\langle a\rangle^{G}$. Then $B$ is a just infinite $Z H$-module where $H=G / A$.

PROOF. Since $G$ is a non-monolithic group, $B$ satisfies the condition (JI 2). Let $C$ be a $G$-invariant subgroup of $B$ (that is $C$ is a $Z H$-submodule of $B$ ), $C \neq\langle 1\rangle$. Then $G / C$ is hypercentral. Lemma 2.1 implies that $G / A$ is a torsion-free nilpotent group of finite 0 -rank. By Lemma 1.3 the group $G$ possesses a finite subnormal series $A=H_{0}<H_{1}<H_{2}<\cdots<H_{n}=G$ such that $H_{1} / H_{0}$ is finitely generated and $H_{i+1} / H_{i}$ are torsion and p-divisible, $1 \leq i \leq n-1$.

Put $B_{1} / C=\langle a\rangle^{H_{1}} C / C, B_{2} / C=\langle a\rangle^{H_{2}} C / C$. Since $H_{1} / A$ is finitely generated, $H_{1}=F_{1} \cdot A$ for some finitely generated subgroup $F_{1}$. Since $G / C$ is a hypercentral group, its finitely generated subgroup $\left\langle a C, F_{1} C / C\right\rangle$ is nilpotent. Since the torsion part of a finitely generated nilpotent group is finite, $B_{1} / C$ is finite. [7, Lemma 5] implies that every $H_{1}$-invariant subgroup of $B_{2} / C$ is $H_{2}$ - invariant. This means that $B_{2} / C=B_{1} / C$, in particular, $B_{2} / C$ is finite. By [7, Lemma 5] after finitely many steps, we obtain the equation $B / C=B_{1} / C$. So, $B / C$ is finite. Hence $B$ satisfies the condition (JI 1), and so $B$ is a just infinite $Z H$-submodule. 
PROPOSITION 2.4. Let $G$ be a non-monolithic group, all proper factor-groups of which are hypercentral groups of finite 0-rank, and let $A$ be a non-identity maximal normal abelian subgroup of $G$. If $A$ is not torsion-free, then $G$ is hypercentral.

ProOF. Assume the contrary. Let $G$ be non-hypercentral, that is $G$ be just-nonhypercentral. Lemma 1.2 implies that $A$ is an elementary abelian $p$-subgroup for some prime $p$. By Lemma $2.1 G / A$ is a nilpotent torsion-free group of finite 0 -rank.

Let $x A \neq A, a A \in \zeta(G / A), 1 \neq a \in A, B=\langle a\rangle$. It follows from Lemma 2.2 that $C_{G}(B)=A$. By Lemma $2.3 B$ is a just infinite $F_{p} H$-module where $H=G / A$. We can consider $B$ as a $J H$-module where $J=F_{p}\langle x\rangle$. By Lemma $2.2 B$ is $J$-torsion-free. From [6, Theorem 2'] we obtain that $H$ is finitely generated and abelian-by-finite, and $B$ is a $J$-minimax module, that is $B$ includes a finitely generated submodule $C$ such that $B / C$ is a $J$-torsion module with the finite set $\Pi_{J}(B / C)$. Since Spec $J$ is infinite, there exists a maximal ideal $P$ such that $P \notin \Pi_{J}(B / C)$. Again $P=y J$ where $y$ is an irreducible polynomial. We can choose $P$ such that deg $y \geq 2$. In particular $C / C P$ is the $P$-component of $B / C P$, hence $B / C P=C / C P \oplus E / C P$, where $E / C P \cong B / C$. It follows that $B P \leq E$, in particular, $B_{1}=B P \neq B$. Since $x A \in \zeta(G / A), B_{1}$ is a $G$-invariant subgroup of $B$. This means that $B / B_{1}$ is finite, $B / B P$ is a vector space over the field $J / P=F_{1}$, so that $B / B_{1}=M_{1} / B_{1} \times \cdots \times M_{k} / B_{1}$ where $M_{i} / B_{1}$ is a minimal $J$-submodule $1 \leq i \leq k$. From the choice of $P$, it follows that $\left|M_{i} / B_{1}\right| \geq p^{2}$ for any $i$. Since $B_{1} \neq\langle 1\rangle, G / B_{1}$ is hypercentral. Then $\zeta\left(G / B_{1}\right) \cap B / B_{1} \neq\langle 1\rangle$. Since $M_{i} / B_{1}$ is a minimal $\langle x\rangle$-invariant subgroup and $x A \in \zeta(G / A)$, either $M_{i} / B_{1} \leq$ $\zeta\left(G / B_{1}\right)$ or $M_{i} / B_{1} \cap \zeta\left(G / B_{1}\right)=\langle 1\rangle$. It follows that there is an index $t$ such that $M_{t} / B_{1} \leq \zeta\left(G / B_{1}\right)$. Since $c B_{1} \in \zeta\left(G / B_{1}\right),\left|\langle c)^{G} B_{1} / B_{1}\right|=p$. On the other hand $\left|\langle c\rangle^{\langle x\rangle} B_{1} / B_{1}\right|=\left|M_{t} / B_{1}\right| \geq p^{2}$. This contradiction shows that $G$ is hypercentral.

\section{Non-monolithic case of characteristic 0}

Everywhere in this section (except Proposition 3.5) $G$ is a just-non-hypercentral non-monolithic group, and $A$ is a maximal normal abelian subgroup of $G$. We assume that $A$ is torsion-free.

Put $\mathscr{P}_{G}(A)=\{B \mid B$ is a non-identity $G$-invariant pure subgroup of $A\}$. We have the following two possibilities: $\cap \mathscr{P}_{G}(A)=\langle 1\rangle$ and $\cap \mathscr{P}_{G}(A) \neq\langle 1\rangle$. Consider the first possibility.

LEMMA 3.1. If $\cap \mathscr{P}_{G}(A)=\langle 1\rangle$, then $G / A$ is torsion-free.

PROOF. Let $T / A$ be the torsion part of $G / A$. Suppose that $T / A \neq\langle 1\rangle$. Then $T / A \cap \zeta(G / A) \neq\langle 1\rangle$. Therefore $T$ contains an element $x \notin A$ such that $x^{p}(A$ for some prime $p$ and $x A \in \zeta(G / A)$. Let $V=\langle x, A\rangle, B \in \mathscr{P}_{G}(A)$. Since $B \neq\langle 1\rangle$ 
then $G / B$ is a hypercentral group. If $V / B$ is torsion-free, $V / B$ is abelian (see, for example, [8, Chapter 66]). Suppose that $V / B$ contains elements of finite order. Let $Y / B$ be the torsion part of $V / B$. Since $A / B$ is torsion-free, $|Y / B|=p$. Then $Y / B$ is normal in $G / B$. Since $Y / B \cap A / B=\langle 1\rangle,[Y, A] \leq B$. So, $V / B=Y / B \times A / B$ is abelian. Hence in each case $V / B$ is abelian. In other words, $[V, V] \leq B$. Since it is true for every subgroup $B \in \mathscr{P}_{G}(A)$, it follows that $[V, V] \leq \cap \mathscr{P}_{G}(A)=\langle 1\rangle$. Consequently, $V$ is abelian. This is a contradiction with the choice of $A$. Hence $G / A$ is torsion-free.

DEFINITION. Let $R$ be a ring, $H$ be a group, and let $A$ be an $R H$-module. We say that $A$ is an $R H$-hypercentral (or $R H$-hypertrivial) module if $A$ has an ascending series of $R H$-submodules

$$
\langle 0\rangle=A_{0} \leq A_{1} \leq \cdots \leq A_{\alpha} \leq A_{\alpha+1} \leq \cdots \leq A_{\gamma}=A
$$

such that $A_{\alpha+1}(x-1) \leq A_{\alpha}$ for every $x \in H, \alpha<\gamma$.

Let $G$ be a just-non-hypercentral group, $A$ be a non-identity normal abelian subgroup of $G$, and $H=G / C_{G}(A)$. Suppose that $A$ is torsion-free. We will consider $A$ as $Z H$-module. Let $D=A \otimes_{Z} Q$. We can extend the action of $H$ on $A$ to the action of $H$ on $D$ in only one way. Let $E$ be a non-zero $Q H$-submodule of $D$, then $E_{1}=E \cap A \neq\langle 0\rangle$. From the relations $A / E_{1}=A / A \cap E \cong A+E / E \leq D / E$, we obtain that $A / E_{1}$ is $Z$-torsion-free. The factor-group $G / E_{1}$ is hypercentral, therefore the $Z H$-module $A / E_{1}$ is $Z H$-hypercentral. Let $E_{1}=C_{0} \leq C_{1} \leq \cdots \leq C_{\alpha} \leq C_{\alpha+1} \leq$ $\cdots \leq C_{\gamma}=A$ be an ascending series of $Z H$-submodules such that $A_{\alpha+1}(x-1) \leq A_{\alpha}$ for each $x \in H, 0 \leq \alpha<\gamma$. Since $A / E_{1}$ is $Z$-torsion-free, we can choose the submodule $C_{\alpha}$ such that $C_{\alpha}$ is pure, $\alpha<\gamma$. Put $Z_{\alpha}=C_{\alpha} \otimes_{Z} Q$. Then, obviously, the series $E=Z_{0} \leq Z_{1} \leq \cdots \leq Z_{\alpha} \leq Z_{\alpha+1} \leq \cdots \leq Z_{\gamma}=D$ is a $Q H$-hypercentral series of $D / E$. Consequently, every proper factor-module of $Q H$-module $D$ is $Q H$ hypercentral. Hence we come to the problem of studying the $Q H$-module $D$, every $Q H$-factor-module of which is $Q H$-hypercentral, where $H$ is a hypercentral group.

Suppose that $\cap \mathscr{P}_{G}(A)=\langle 0\rangle$. Let $L=\cap_{B \in T} B \otimes_{Z} Q$, where $T=\mathscr{R}_{G}(A)$ If we assume that $L \neq\langle 0\rangle$ then $L_{1}=L \cap A \neq\langle 0\rangle$. On the other hand,

$$
L \cap A=\left(\cap_{B \in T} B \otimes_{Z} Q\right) \cap A=\cap_{B \in T}\left(B \otimes_{Z} Q \cap A\right)=\cap_{B \in T} B=\langle 0\rangle .
$$

This means that $L=\langle 0\rangle$ and therefore $D$ is a non-monolithic $Q H$-module.

PROPOSITION 3.2. Let $H$ be a hypercentral torsion-free group, $D$ a non-monolithic $Q H$-module, and $C_{H}(D)=\langle 1\rangle$. Suppose that every proper factor-module of $D$ is QH-hypercentral.

(1) If $1 \neq x \in \zeta(H)$, then $D$ is $Q\langle x\rangle$-torsion-free. 
(2) If $H$ has finite 0 -rank, then $D$ is $Q H$-hypercentral .

PROOF. (1) Let $T$ be the $Q\langle x\rangle$-torsion part of $D$. Suppose that $T \neq\langle 0\rangle$. Since $x \in \zeta(H)$, the $I$-component of $D$ is a $Q H$-submodule for every ideal $I$ of ring $Q\langle x\rangle$. It follows that $\Pi_{Q\langle x\rangle}(T)=\{P\}$ for some maximal ideal $P$ of $Q\langle x\rangle$. Put $T_{1}=\{a \in$ $T \mid a P=\langle 0\rangle\}$. Assume that $T \neq T_{1}$. Then $D / T_{1}$ is a $Q H$-hypercentral module. Thus for every element $d \in D$, there is a number $n \in \mathbf{N}$ such that $d(x-1)^{n} \in T_{1}$. Since $\Pi_{Q\langle x\rangle}\left(T / T_{1}\right)=\{P\}$, this means that $P=(x-1) Q\langle x\rangle$. But in this case $T_{1} \leq C_{D}(x)$; that is $C_{D}(x) \neq\langle 0\rangle$. This is a contradiction of Lemma 1.2. Hence $T=T_{1}$. Put $C=D P$, then $T \cap C=\langle 0\rangle$. This means that $C=\langle 0\rangle$; that is $D=T_{1}$. Since $D$ is non-monolithic, $D$ includes a proper non-zero $Q H$-submodule $E$. Then $D / E$ is a $Q H$-hypercentral torsion module with $\Pi_{Q\langle x\rangle}(D / E)=\{P\}$. It follows that $P=(x-1) Q\langle x\rangle$, which is impossible, and so (1) is proved.

(2) Assume that $D$ is non- $Q H$-hypercentral. Put $J=Q\langle x\rangle$. We can consider $D$ as $J H$-module. Let $0 \neq d \in D, E=d J H$, and $\pi=\{P \mid P \in \operatorname{Spec}(J)$ and $E \neq E P\}$ :

Since $E$ is not $J$-torsion, [20, Theorem 2.3] implies that the set $\pi$ is infinite. Thus $\pi$ contains an ideal $P$ such that $P \neq J(x-1)$. From the choice of $x$, we obtain that $E P$ is a $Q H$-submodule. It follows that $D / E P$ is a $Q H$-hypercentral module. In particular, $\zeta_{Q H}(D / E P) \cap E / E P=L / E P \neq\langle 0\rangle$. This means that $L(x-1) \leq E P$. On the other hand, $L P \leq E P$. Since $P$ and $J(x-1)$ are distinct maximal ideals of $J, P+J(x-1)=J$. From the inclusions $L(x-1) \leq E P, L P \leq E P$, we obtain that $L \leq E P$, in particular, $L / E P=\langle 0\rangle$. This contradiction proves that $D$ is $Q H$-hypercentral.

Consideration of the case when $\cap \mathscr{P}_{G}(A) \neq\langle 1\rangle$ is our next step.

LEMMA 3.3. If $\cap \mathscr{P}_{G}(A) \neq\langle 1\rangle$ then $\cap \mathscr{P}_{G}(A)=A$.

Proof. Assume the contrary, and let $B=\cap \mathscr{P}_{G}(A) \neq\{A\}$. Then $B$ is a proper $G$-invariant pure subgroup of $A$. Lemma 1.2 yields that $A=C_{G}(A)$. Put $H=G / A$. We will consider $A$ as a $Z H$-module. Put $D=A \otimes_{Z} Q$. We can consider $D$ as a $Q H$-module. Let $E=B \otimes_{z} Q$, then $E$ is a proper $Q H$-submodule of $D$. If $C$ is a proper $G$-invariant non-identity subgroup of $B$, then from the choice of $B$ we obtain that $B / C$ is a torsion group. It follows that $E$ is a simple $Q H$-submodule. Since $E \neq\langle 0\rangle$, the factor-module $D / E$ is $Q H$-hypercentral. Let $V / E=\zeta_{Q H}(D / E)$. By Lemma 1.4 there exists a $Q H$-submodule W such that $V=E \oplus W$. It follows from the choice of $D$ that $W_{1}=V \cap A \neq\langle 0\rangle$. Hence $W_{1}$ is a non-identity $G$-invariant subgroup of $A$ such that $B \cap W_{1}=\langle 1\rangle$. This is a contradiction of Lemma 1.1. So, $\mathscr{P}_{G}(A)=\{A\}$. 
PROPOSITION 3.4. Let $G$ be a non-monolithic group, the proper factor-groups of which are hypercentral groups of finite 0-rank, and let $A$ be maximal normal abelian subgroup of $A$. If $A$ is a non-identity torsion-free subgroup, then $G$ is hypercentral.

PROOF. If $\cap \mathscr{P}_{G}(A)=\langle 1\rangle$, then we can use Proposition 3.2. Suppose that $\cap \mathscr{P}_{G}(A) \neq\langle 1\rangle$. Assume that $G$ is not-hypercentral. Lemma 3.3 implies that for every non-identity $G$-invariant subgroup $B$ of $A$, the factor-group $A / B$ is torsion.

Let $1 \neq a \in A, B=\langle a\rangle^{G}, \pi=\left\{p \mid p\right.$ is a prime such that $\left.B \neq B^{p}\right\}$. [20, Theorem 2.3] proves that the set $\pi$ is infinite. Let $p \in \pi$. Since $B / B^{p}=\langle a\rangle^{G} B^{p} / B^{p}, B / B^{p}$ includes a proper $G$-invariant maximal subgroup $M_{p} / B^{p}$. Since $G / B^{p}$ is hypercentral, and any chief factor of a locally nilpotent group is central (see, for example, [13, Theorem 5.27, Corollary 1]), $[B, G] \leq M_{p}$. It follows that $[B, G] \leq \cap_{p \in \pi} M_{p}$. If $[B, G] \neq\langle 1\rangle$ then the factor-group $B /[B, G]$ is torsion. Since $\pi$ is infinite, the set $\Pi(B /[B, G])$ is infinite too. On the other hand, $B /[B, G]=\langle a\rangle^{C}[B, G] /[B, G]=$ $\langle a\rangle[B, G] /[B, G]$. Hence $B /[B, G]$ is finite. This contradiction shows that $[B, G]=$ $\langle 1\rangle$, that is $B \leq \zeta(G)$. But this is a contradiction of Lemma 1.2. Consequently, $G$ is hypercentral.

PROOF OF THEOREM 1. Let $A$ be a maximal normal abelian subgroup of $G$. Since Fitt $G \neq\langle 1\rangle, A \neq\langle 1\rangle$. If $A$ is not torsion-free then $G$ is hypercentral by Proposition 2.4. If $A$ is torsion-free, then $G$ is hypercentral by Proposition 3.4.

\section{Monolithic case}

LEMMA 4.1. Let $G$ be a monolithic just-non-hypercentral group and let $M$ be the monolith of $G$. If $M$ is abelian, then $M$ is a maximal normal abelian subgroup of $G$; in particular, $M=C_{G}(M)$. Moreover, $M=\operatorname{Fitt} G$.

PROOF. Let $A$ be a maximal normal abelian subgroup of $G$ such that $M \leq A$. Suppose that $A \neq M$. Lemma 1.2 implies that either $A$ is an elementary abelian $p$-subgroup for some prime $p$, or $A$ is torsion-free. Consider the first case. Since $G / M$ is hypercentral, $\langle 1\rangle \neq A / M \cap \zeta(G / M)$. Let $a M \neq M, a M \in \zeta(G / M) \cap A / M$, $B=\langle a, M\rangle$. We can consider $B$ as $F_{p} H$-module, where $H=G / A$. Then $M$ is a simple $F_{p} H$-submodule of $B$, and $[B, g] \leq M$ for any $g \in G$. By Lemma 1.4 there exists a $G$-invariant subgroup $C$ such that $M \cap C=\langle 1\rangle$. This contradicts Lemma 1.1.

Let $A$ be a torsion-free subgroup. We can consider $A$ as $Z H$-module. Put $D=$ $A \otimes_{Z} Q$. We can consider $D$ as $Q H$-module. Since $M$ is a simple $Z H$-module, the additive group of $M$ is divisible, and $M=M \otimes_{Z} Q$. Since $M$ is divisible, $A=M \times U$ for some subgroup $U$ (see, for example, [3, Theorem 21.2]). This means that $A / M$ is torsion-free. Since $G / M$ is hypercentral , $\zeta(G / M) \cap A / M$ is non-trivial. Let 
$a M \neq M, a M \in \zeta(G / M) \cap A / M, E=\langle a, M\rangle \otimes_{Z} Q$. Then $E / M$ is a $Q H$-central factor of $Q H$-module $D$. By Lemma 1.4 there exists a $Q H$-submodule $C$ such that $E=M \oplus C$. It follows from the choice of $D$ that $C_{1}=C \cap A=\langle 1\rangle$. But in this case $C_{1} \cap M=\langle 1\rangle$, and we obtain a contradiction of Lemma 1.1.

Put $F=$ Fitt $G$, and assume that $M \neq F$. Since $G / M$ is hypercentral, $F / M \cap$ $\zeta(G / M) \neq\langle 1\rangle$. Let $M \neq x M \in \zeta(G / M) \cap F / M, 1 \neq a \in M$. The subgroup $L=\langle x, a\rangle$ is nilpotent (see, for example, [13, Theorem 2.18]). It follows that $C_{M \cap L}(x) \neq\langle 1\rangle$. However this is in contradiction with Lemma 1.2. Hence $M=$ $F$.

LEMMA 4.2. Let $H$ be a hypercentral group, $M$ a simple $Z H$-module, $C_{H}(M)=$ $\langle 1\rangle, C=\zeta(H)$, and let $T$ be the torsion part of $C$.

(1) If $M$ is $Z$-torsion-free, then $T$ is a locally cyclic subgroup.

(2) If $M$ is an elementary abelian p-subgroup for some prime $p$, then $T$ is a locally cyclic $p^{\prime}$-subgroup.

(3) If $H$ has finite 0-rank, then $M$ is an elementary abelian p-subgroup for some prime $p$, and $C$ is a locally cyclic $p^{\prime}$-subgroup.

Proof. Put $E=\operatorname{End}_{Z H}(M)$. Then $E$ is a divisible algebra by Schur's theorem. Let $Z$ be the center of $E$. Then $Z$ is a subfield of $E$. For every element $c \in C$ the mapping $\tau_{c}: a \rightarrow a c, a \in M$, is a $Z H$-automorphism of $M$, and the mapping $v: c \rightarrow \tau_{c}, c \in C$, is an imbedding of $C$ in the multiplicative group of $Z$ because $C_{H}(M)=\langle 1\rangle$. It follows from [4, Theorem 127.3] that $T$ is a locally cyclic subgroup (moreover, it is a $p^{\prime}$-subgroup if $M$ is an elementary abelian $p$-group). If $r_{0}(H)$ is finite, then $A$ is an elementary abelian $p$-group for some prime $p$ by [20, Theorem 2.3]. From [20, Theorem 2.3], we obtain that $C$ is a torsion subgroup.

PROOF OF THEOREM 2. Lemma 4.1 implies that $M$ is the hypercentral residual of $G$. It follows from [19, Theorem $2^{\prime}$ ] that $G$ includes a subgroup $H$ such that $G$ is a split extension of $M$ by $H$, and $H=N_{G}(H)$ is hypercentral. By [19, Theorem 2'] all complements to $M$ are conjugate. Condition (1) follows from Lemma 4.1, condition (2) follows from Lemma 4.1. Conditions (5) and (6) follow from Lemma 4.2.

The last statement of Theorem 2 follows from previous statements and Lemma 4.2.

The question about the existence of groups from Theorems 1 and 2 is natural. The following theorem clarifies this situation.

THEOREM 3. Let $H$ be a hypercentral group, $C=\zeta(H)$, and let $T$ be the periodic part of $C$.

(1) If $T=C$ is a locally cyclic $p^{\prime}$-subgroup, and $p$ is prime, then there exists a simple $F_{p} H$-module $M$ such that $C_{H}(M)=\langle 1\rangle$. 
(2) If $H$ has infinite 0-rank, and $T$ is a locally cyclic group, then there exists a simple $Z H$-module $M$ such that $C_{H}(M)=\langle 1\rangle$ and the additive group of $M$ is torsion-free.

(3) If $H$ has infinite 0-rank, and $T$ is a locally cyclic $p^{\prime}$-subgroup for some prime $p$, then there exists a simple $F_{p} H$-module $M$ such that $C_{H}(M)=\langle 1\rangle$.

ProOF. (1) There exists a simple $F_{p} C$-module $B$ such that $C_{C}(B)=\langle 1\rangle$ (see, for example, [17, Section 4]). Consider the $F_{p} H$-module $B^{*}=B \otimes_{F_{p} C} F_{p} H$ and identify, in the natural way, $B$ with the $F_{p} C$-submodule of $B \otimes 1$. Then $B^{*}=\oplus_{t \in Y} B t$ where $Y$ is the transversal to $C$ in $H$. Let $M$ be a $F_{p} H$-composition factor of $B^{*}$. Then $M$ is a simple $F_{p} H$-module. Since $B^{*}$ is a semisimple $F_{p} C$-module, there exists a non-empty subset $S$ of $Y$ such that $M$ is isomorphic to $M_{0}=\oplus_{t \in S} B t$. If $t \in S$ then $C_{C}(M) \leq C_{C}(B t)=t^{-1} C_{C}(B) t=C_{C}(B)$. This means that $C_{C}(M)=\langle 1\rangle$. Hence $C_{H}(M)=\langle 1\rangle$.

(2) Since $H$ has an infinite 0 -rank, $H$ includes an abelian subgroup $V$ of infinite 0 -rank (see, for example, [14, Theorem 6.36]). We can assume that $C \leq V$. Let $Q$ be a maximal periodic subgroup of $V$ with the property $T \cap Q=\langle 1\rangle$, and let $T_{1} / Q$ be the periodic part of $V / Q$. Then $\operatorname{Soc}\left(T_{1} / Q\right)=(\operatorname{Soc} T) Q / Q \cong \operatorname{Soc} T$, in particular, $\operatorname{Soc}\left(T_{1} / Q\right)$ is locally cyclic. It follows that $T_{1} / Q$ is locally cyclic. Hence there exists a simple $Z V$-module $B$ such that $C_{V}(B)=Q$ and the additive group of $B$ is torsion-free (see [17, Proposition 4.13]). It follows from the choice of $B$ that $C_{V}(B) \cap C=\langle 1\rangle$. Put $B^{*}=B \otimes_{\mathrm{z} C} Z H$, then $B^{*}=\oplus_{t \in S} B t$, where $S$ is a transversal to $V$ in $H$. Let $M$ be a composition $Z H$-factor of $B^{*}$, then $M$ is a simple $Z H$-module and $M \cong \oplus_{t \in R} B t$ for some subset $R$ of $S$. For every $t \in R$, we have $C_{H}(M) \cap C \leq C_{C}(B t)=t^{-1} C_{C}(B) t=\langle 1\rangle$. This means that $C_{H}(M) \cap C=\langle 1\rangle$. Since $H$ is hypercentral, $C_{H}(M)=\langle 1\rangle$. The proof of (3) is similar.

REMARK. Lemma 4.2 shows that if $M$ is a simple $Z H$-module with $C_{H}(M)=\langle 1\rangle$, then $M$ is an elementary abelian $p$-subgroup for some prime $p$ and $C=\zeta(G)$ is a locally cyclic $p^{\prime}$-subgroup. Conversely, Theorem 3 (1) implies that for such group $H$ there exists a simple $F_{p} H$-module $M$ with identity centralizer.

\section{Acknowledgments}

The authors would like to thank the 'Volkswagen-Stifftung' (RIP program in Oberwolfach) for the support of their research. 


\section{References}

[1] S. Franciosi and F. de Giovanni, 'Soluble groups with many Chernikov quotients', Atti Accad. Naz. Lincei Rend. Cl. Sci. Fis. Mat. Natur. 79 (1985), 19-24.

[2] _ - 'Soluble groups with many nilpotent quotients', Proc. Roy. Irish Acad. Sect. A 89 (1989), $43-52$.

[3] L. Fuchs, Infinite abelian groups, vol. 1 (Academic Press, New York, 1973).

[4] - Infinite abelian groups, vol. 2 (Academic Press, New York, 1973).

[5] J. R. J. Groves, 'Soluble groups with every proper quotients polycyclic', Illinois J. Math. 22 (1979), 90-95.

[6] M. J. Karbe and L. A. Kurdachenko, 'Just infinite modules over locally soluble groups', Arch. Math. 51 (1988), 401-411.

[7] L. A. Kurdachenko, 'Locally nilpotent groups with the weak minimal condition for normal subgroups', Siberian Math. J. 25 (1984), 589-594.

[8] A. G. Kurosh, Theory of groups (Nauka, Moscow, 1967).

[9] D. McCarthy, 'Infinite groups whose proper quotients are finite', Comm. Pure Appl. Math. 21 (1968), 545-562.

[10] _ - 'Infinite groups whose proper quotients are finite', Comm. Pure Appl. Math. 23 (1970), 767-789.

[11] M. F. Newmann, 'On a class of metabelian groups', Proc. London Math. Soc. 10 (1960), 354-364.

[12] —_ 'On a class of nilpotent groups', Proc. London Math. Soc. 10 (1960), 365-375.

[13] D. J. S. Robinson, Finitness conditions and generalized soluble groups, Part I (Springer, Berlin, 1972).

[14] - Finitness conditions and generalized soluble groups, Part 2 (Springer, Berlin, 1972).

[15] _ ' 'Groups whose homomorphic images have a transitive normality relation', Trans. Amer. Math. Soc. 176 (1973), 181-213.

[16] D. J. S. Robinson and J. S. Wilson, 'Soluble groups with many polycyclic quotients', Proc. London Math. Soc. 48 (1984), 193-229.

[17] D. J. S. Robinson and Z. Zhang, 'Groups, whose proper quotients have finite derived subgroups', J. Algebra 118 (1988), 346-368.

[18] J. S. Wilson, 'Groups with every proper quotients finite', Math. Proc. Cambridge Phil. Soc. 69 (1971), 373-391.

[19] D. I. Zaitsev, 'Hypercyclic extensions of abelian groups', in: The groups defined by the properties of systems of subgroups (Inst. of Math., Kiev, 1979) pp. 16-37.

[20] - 'Products of abelian groups', Algebra i Logika 19 (1980), 150-172.

[21] _ - 'The residual nilpotence of some metabelian groups', Algebra i Logika 20 (1981), 638-653.

[22] Z. R. Zhang, 'Groups whose proper quotients are finite-by-nilpotent', Arch. Math. 57 (1991), 521-530.

L. A. Kurdachenko

Algebra Department

Dnepropetrovsk University

Provulok Naukovyi 13

320625 Ukraine

e-mail: mmf@ff.dsu.dp.ua
I. Y. Subbotin

Mathematics Department National University 9920 S.La Cienega Blvd Inglewood, CA 90301, USA e-mail: isubboti@nunic.nu.edu 